\title{
Comparison of hazard of death following surgery for colon versus rectal cancer
}

\author{
D S Ediriweera ${ }^{1}$, S Kumarage ${ }^{2}$, K I Deen ${ }^{2}$ \\ (Index words: colon cancer, rectal cancer, survival, hazard of death)
}

\begin{abstract}
Introduction Prognosis in cancer is usually assessed by use of Kaplan-Meier survival function estimate curves, which reflect survival, or the proportion of patients that will remain alive after a particular event at a given time. By contrast, hazard function represents the proportion expected to be deceased among those surviving at a given time after an event.
\end{abstract}

Objectives To evaluate survival and hazard of death, in patients with colon cancer (CC) and rectal cancer (RC), as indices of prognosis.

Methods Colon and rectal cancer patients who underwent surgical resection with curative intent from 1996 to 2011 were studied. The hazard of death and survival patterns were assessed with Weibull Hazard models and KaplanMeier survival function estimate curves.

Results There were $119 \mathrm{CC}$ and $250 \mathrm{RC}$ patients included in the study. Median (Inter-quartile range: IQR) age of both groups was $58(49-66.5)$ years. The median (IQR) followup time was 30 (12 - 72) months for CC and $30(13-70)$ months for RC. Both groups were similar in comparison with regard to age $(p=0.96)$, gender $(p=0.56)$, tumour stage $(p=0.33)$, vascular invasion $(p=0.69)$, lymphatic invasion $(p=0.33)$, perineural invasion $(p=0.94)$, degree of tumour differentiation $(p=0.38)$ and preoperative carcinoembryonic antigen levels $(p=0.77)$. CC showed better overall survival compared to RC $(p=0.03)$ with a 5 -year survival rate of $72 \%$ versus $60 \%$ respectively. After curative resection, CC showed a $6 \%$ decrease in hazard of death with time compared with RC which showed a $1 \%$ increase in the hazard of death with time.

Conclusions Among patients who underwent resectional surgery, CC had a better prognosis than RC.

Ceylon Medical Journal 2016; 61: 52-55

DOI: http://doi.org/10.4038/cmj.v61i2.8285

\section{Introduction}

Globally, cancers account for $13 \%$ of all reported deaths [1]. Annually, 1.2 million new colorectal cancers are expected worldwide - this has become the second most common cancer in women and third commonest in men [2]. Furthermore, colorectal cancer accounts for 600000 deaths worldwide annually [2-4]. The literature indicates that survival in patients with colorectal cancervaries by the site of tumour [5, 6], and previous survival comparisons show that colon cancer has better survival compared to rectal cancer [7]. Differences in survival for colon versus rectal cancer have been reported in relation to gender, age, body mass index, alcohol consumption, histological characteristics, differences in molecular patterns and gene expression [8-10]. Most studies have focused on survival as a prognostic index after curative resection for cancer. This study was designed to compare survival in colon and rectal cancer and to assess the hazard of death with time, for colon and rectal cancer in a Sri Lankan population.

\section{Methods}

All patients diagnosed with colorectal cancer and who underwent surgical resection at the University Surgical Unit, North Colombo Teaching Hospital from 1996 to 2011 were studied. Cancer related mortality was included in analysis and we excluded post-operative deaths within 30 days of operation. Surgical resections were performed by the same team according to a standard protocol. Demographic data, investigation results, treatment modalities, and tumour histopathologic features of the study population are shown in Tables 1 and 2.

Large bowel cancer from caecum to the recto-sigmoid junction was classified as colon cancer (CC) and the rest was classified as rectal cancer (RC). Resection of colon cancer included total mesocolic excision with en bloc

${ }^{1}$ ICT Centre and ${ }^{2}$ Department of Surgery, Faculty of Medicine, University of Kelaniya, Sri Lanka.

Correspondence: DSE, e-mail: <dileepa@kIn.ac.lk>. Received 21January and revised version accepted 16 March 2016. 
resection of an adjacent organ if there was tumour attachment at the time of surgery. For rectal cancer, we performed mesorectal excision to a distance of $5 \mathrm{~cm}$ distal to the lower limit in tumours located higher than $10 \mathrm{~cm}$ from the anal verge. Total mesorectal excision was done for rectal cancers located between 0 and $10 \mathrm{~cm}$ from the anal verge. The majority received long-course preoperative chemoradiation followed by surgical resection as described previously [11]. Tumour staging was according to the American Joint Committee on Cancer (AJCC) TNM staging system, 6th edition [12]. All patients were followed up at the surgical clinic. Patients who failed to attend clinic or could not be contacted for more than a year were considered as lost to follow up. The study was approved by the Ethical Review Committee of Faculty of Medicine, University of Kelaniya, Sri Lanka.

Right censored CC and RC survival data were used in analysis [13]. Follow up time was defined as the time between surgery to death, or date at which the patient was last confirmed to be alive (censoring time). Data were analysed using Kaplan-Meier survival function estimate curves [14] and Weibull Hazard models [15]. Kaplan-Meier survival function estimate curves were used to estimate survival rates and Weibull Hazard models were used to assess the significance of cancer location as a survival determinant in colorectal patients and to estimate hazard function (i.e. hazard of death in the present study) for total follow up time of colon and rectal cancer. Hazard function represents the proportion expected to die (or the immediate mortality risk) among those surviving at a given time after curative resection [16] and survival rate reflects the proportion of patients remaining alive from a specific disease (i.e. colon and rectal cancer in the present study) at a given time after curative resection [17]. In this study, both hazard of death and estimation of survival were assessed to explain the prognosis of $\mathrm{CC}$ and $\mathrm{RC}$ after curative resection.

Comparison of groups wasundertaken using Pearson's Chi-square test, Fisher exact test and Mann Whitney $\mathrm{U}$ test as appropriate. A $p$ value $<0.05$ was considered significant. Analysis was done using the SAS System (version 9.0; Cary, USA) [18].

Table 1. Comparison of demography and pathological features in patients with colon $(n=119)$ and rectal $(n=250)$ cancer

\begin{tabular}{|c|c|c|c|c|}
\hline & & $\begin{array}{c}\text { Colon cancer } \\
M^{*}\left(I Q R^{\dagger}\right) \text { or } N^{*}(\%)\end{array}$ & $\begin{array}{c}\text { Rectal cancer } \\
M(I Q R) \text { or } N(\%)\end{array}$ & $p$ value ${ }^{\mathrm{a}}$ \\
\hline \multicolumn{2}{|l|}{ Age (in years) } & $58(47-68)$ & $58(49-66)$ & 0.96 \\
\hline \multicolumn{2}{|l|}{ Male gender } & $58(49 \%)$ & $130(52 \%)$ & 0.56 \\
\hline \multicolumn{2}{|l|}{ Adenocarcinoma } & $108(91 \%)$ & $244(98 \%)$ & $<0.01$ \\
\hline \multicolumn{2}{|l|}{ Mucinous } & $11(09 \%)$ & $6(02 \%)$ & \\
\hline \multirow[t]{3}{*}{ Differentiation } & Well & $26(22 \%)$ & $43(17 \%)$ & 0.38 \\
\hline & Moderate & $85(71 \%)$ & $195(78 \%)$ & \\
\hline & Poor & $8(07 \%)$ & $12(05 \%)$ & \\
\hline \multirow[t]{4}{*}{ AJCC Stage } & I & $20(17 \%)$ & $57(23 \%)$ & 0.33 \\
\hline & II & $45(38 \%)$ & $78(31 \%)$ & \\
\hline & III & $47(39 \%)$ & $93(37 \%)$ & \\
\hline & IV & $7(06 \%)$ & $22(09 \%)$ & \\
\hline \multicolumn{2}{|l|}{ Vascular invasion } & $9(08 \%)$ & $22(09 \%)$ & 0.69 \\
\hline \multicolumn{2}{|c|}{ Lymphatic invasion } & $9(08 \%)$ & $27(11 \%)$ & 0.33 \\
\hline \multicolumn{2}{|c|}{ Peri-neural invasion } & $13(11 \%)$ & $28(11 \%)$ & 0.94 \\
\hline \multicolumn{2}{|c|}{ Positive resection margin } & $1(01 \%)$ & $30(12 \%)$ & $<0.01$ \\
\hline \multicolumn{2}{|c|}{ Lymph node harvest } & $10(7-16)$ & $8(4-12)$ & $<0.01$ \\
\hline \multicolumn{2}{|c|}{ Preoperative CEA level(ng/ml) } & $6(2.5-11.5)$ & $5(2.5-15)$ & 0.77 \\
\hline
\end{tabular}

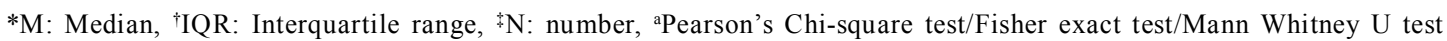


Table 2. Type of surgical procedure

\begin{tabular}{lc}
\hline & Number \\
\hline Right hemicolectomy & 43 \\
Left hemicolectomy & 18 \\
Sigmoid colectomy & 37 \\
Transverse colectomy & 5 \\
Subtotal colectomy & 18 \\
Total colectomy & 3 \\
Restorative proctocolectomy & 9 \\
Anterior resection & 179 \\
Abdomino-perineal resection & 27 \\
Emergency surgery for colon cancer* & 6 \\
Emergency surgery for rectal cancer* & 24 \\
\hline
\end{tabular}

*Hartmann's procedure or Paul-Mickulicz procedure

\section{Results}

Of 369 patients, 119 (32\%) had CC and $250(68 \%)$ had RC. One hundred eighty eight (51\%) were men (Table 1). The entire study population had undergone surgical resection and details of surgical procedures are given in Table 2. Of these, $338(99 \% \mathrm{CC}$ and $88 \% \mathrm{RC})$ were margin negative $\left(R_{0}\right)$ resections and $31(1 \% \mathrm{CC}$ and $12 \% \mathrm{RC})$ were margin positive $\left(R_{1}\right)$ resections. The mean follow up time after surgery was 47 months (median, 30 months; interquartile range, 12 - 72 months) for $\mathrm{CC}$ and 45 months (median, 30 months; inter-quartile range, 13 - 70 months) for RC. Colon and rectal cancer groups were similar in terms of gender $(p=0.56)$, age $(p=0.96)$, tumour stage $(p=0.33)$, vascular invasion $(p=0.69)$, lymphatic invasion $(p=0.33)$, perineural invasion $(p=0.94)$, degree of tumour differentiation $(p=0.38)$ and pre-operative carcinoembryonic antigen (CEA) levels $(p=0.77)$.

Weibull hazard model analysis revealed that cancer location (colonic or rectal) was a significant determinant of survival for colorectal cancer $(p=0.03$ ) which continued to remain significant after adjustment for relevant cancer stages $(p=0.04)$. Comparison of average survival at any time point showed that survival for rectal cancer was 58\% that of colon cancer (i.e. parameter estimate for cancer location after adjusting for cancer stage, $\beta=0.58$ ). KaplanMeier survival function estimate curves revealed that $\mathrm{CC}$ had better survival compared to RC (Figure 1, Log rank $p=0.03$ ) and the rate of 5-year (actuarial) survival for colon versus rectal cancer was $72 \%$ and $60 \%$ respectively.

After curative resection, we observed that the overall average hazard of death for colon cancer was lower than for rectal cancer. Furthermore, the hazard of death for $\mathrm{CC}$ progressively decreased from the post-operative period up to the time of last follow up, and revealed that the risk of death at 10 years after resection for colon cancer was virtually zero. Thus, for colon cancer, the overall hazard of death had decreased following curative resection by an average of $0.6 \%$. On the other hand, for rectal cancer, the hazard of death had only decreased until the eighth postoperative year. Thereafter, hazard of death showed an increase with time, resulting in an overall increase in the hazard of death from the time of surgery by $0.1 \%$ (i.e. estimated scale parameter, $\sigma=0.9$ ) (Figure 2). The increasing trend in hazard of death was also associated with the negative resection margin RC patients who continued to live more than 8 years post-operatively. Rectal cancers with a positive resection margin showed a higher hazard of death, until the last patient with a positive margin died six and half years after surgery (Figure 3).

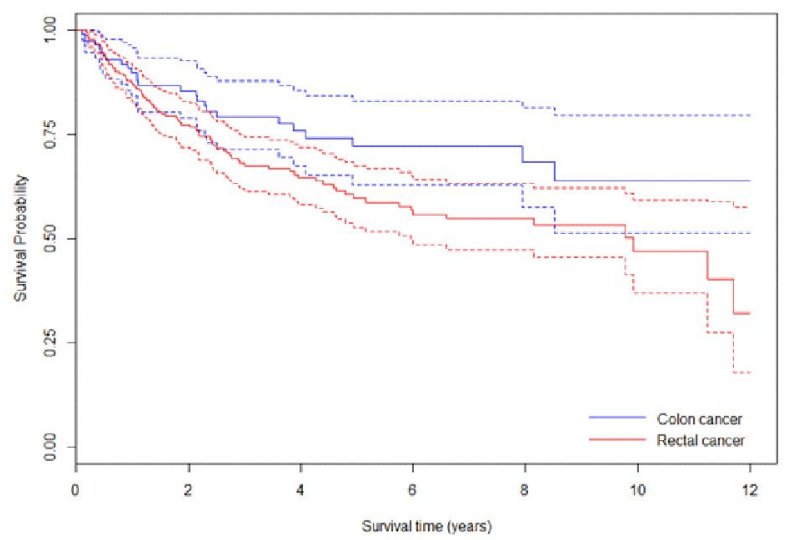

Figure 1. Comparison of survival for colon and rectal cancer.

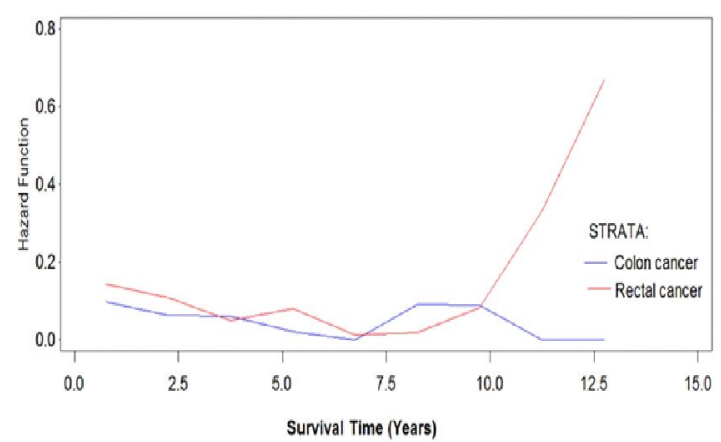

Figure 2. Hazard of death for colon and rectal cancer.

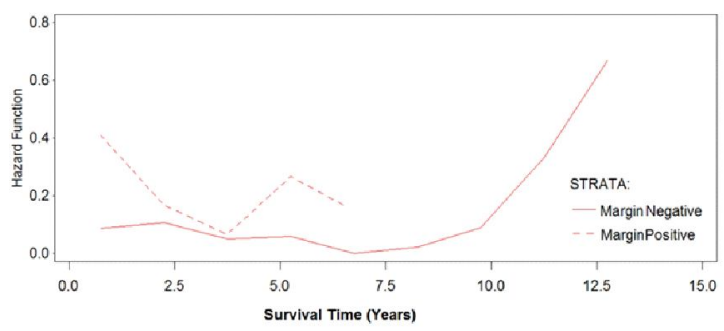

Figure 3. Hazard of death for margin positive and margin negative rectal cancer. 


\section{Discussion}

In this study we found that the overall hazard of death decreased after curative resection for colon cancer, whereas for rectal cancer, there was an increase in the overall hazard of death after curative resection, indicating that rectal cancer had a worse prognosis compared with colon cancer. Since the hazard of death for patients with margin negative rectal cancer decreased, at least in the first six post-operative years, selective protocol based chemo-radiation and surgery is useful in rectal cancer.

Cancer location was a significant survival determinant in colorectal cancer, so that colon cancer showed a better survival rate compared to rectal cancer, and on average, survival of patients with $\mathrm{RC}$ was $60 \%$ that of $\mathrm{CC}$, which underscores the importance of early diagnosis and treatment in rectal cancer to achieve an improved prognosis. Although the number of patients in this study is sufficient to demonstrate statistically significant differences, data from other studies are required to further strengthen our conclusions.

\section{Conflicts of interests}

There are no conflicts of interest.

\section{References}

1. Cancer, Fact sheet. World Health Organization, 2015. http://www.who.int/mediacentre/factsheets/fs297/en/ (accessed January 16, 2015)

2. Ferlay J, Shin HR, Bray F, et al. Estimates of worldwide burden of cancer in 2008: GLOBOCAN 2008. Int J Cancer 2010; 127: 2893-17.

3. American Cancer Society. Global Cancer Facts \& Figures 2nd Edition. Atlanta: American Cancer Society, 2011.

4. Akhoond MR, Kazemnejad A, Hajizadeh E, et al. Comparison of colon and rectum cancer: survival and prognostic factors. Gastroenterol Hepatol Bed Bench 2010; 3: $177-86$.

5. Siegel R, Ward E, Brawley O, et al. Cancer statistics, 2011. CA Cancer J Clin 2011; 61:212-36.
6. Jemal A, Bray F, Center MM, et al. Global cancer statistics. CA Cancer J Clin 2011; 61: 69-90.

7. Gatta G, Ciccolallo L, Capocaccia R, et al. Differences in colorectal cancer survival between European and US populations: the importance of sub-site and morphology. Eur J Cancer 2003; 39: 2214-22.

8. Li F, Lai M. Colorectal cancer, one entity or three. J Zhejiang Univ Sci B2009; 10: 219-29.

9. Asghari Jafarabadi M, Mohammadi SM, Hajizadeh E, et al. Does the prognosis of colorectal cancer vary with tumor site? Gastroenterol Hepatol Bed Bench 2011; 4: 199-209.

10. Roncucci L, Fante R, Losi L, et al. Survival for colon and rectal cancer in a population-based cancer registry. Eur $J$ Cancer 1996; 32: 295-302.

11. Wijenayake W, Perera M, Balawardena J, et al. Proximal and distal retal cancers differ in curative resectability and local recurrence. World J Gastrointest Surg 2011; 3: 113-8.

12. Greene FL, Page DL, Fleming ID, et al. Colon and Rectum. AJCC Cancer Staging Manual. 6th edn. New York, Springer. 2002: 113-23.

13. Klein JP, Joel PK. Survival analysis: State of the Art. 1992 edn. Ohio, Springer. 1992: 11-24.

14. The LIFETEST Procedure. Survival Analysis Using SAS $₫$ : A Practical Guide. Second Edition.Cary, NC, SAS Institute. 2010: 29-69.

15. The LIFEREG Procedure. Survival Analysis Using SAS $₫$ : A Practical Guide. Second Edition. Cary, NC, SAS Institute. 2010: 71-124.

16. Schneider, Michael F, Stephen J. Gange, et al. Patterns of the hazard of death after AIDS through the evolution of antiretroviral therapy: 1984-2004. AIDS 2005; 19: 2009-18.

17. Allison, Paul David. Survival analysis using SAS: APractical Guide.Second Edition.Cary, NC, SAS Institute. 2010: $9-28$.

18. SAS Institute, Statistical Analysis System SAS V9. 02004. 